\title{
VSIG4 expression on macrophages facilitates lung cancer development
}

\author{
Yunmei Liao', Sheng Guo ${ }^{2}$, Yongwen Chen², Dayan $\mathrm{Cao}^{2}$, Huan $\mathrm{Xu}^{2}$, Chengying Yang ${ }^{2}$, Lei Fei ${ }^{2}$, Bing $\mathrm{Ni}^{2}$ and \\ Zhihua Ruan ${ }^{1}$
}

Tumor-associated macrophages are a prominent component of lung cancer stroma and contribute to tumor progression. The protein V-set and Ig domain-containing 4 (VSIG4), a novel B7 family-related macrophage protein that has the capacity to inhibit T-cell activation, has a potential role in the development of lung cancer. In this study, 10 human non-small-cell lung cancer specimens were collected and immunohistochemically analyzed for VSIG4 expression. Results showed massive VSIG4 ${ }^{+}$cell infiltration throughout the samples. Immunofluorescent double staining showed that VSIG4 was present on $\mathrm{CD}_{6}{ }^{+}$macrophages, but absent from $\mathrm{CD} 3^{+} \mathrm{T}$ cells, $\mathrm{CD} 31^{+}$endothelial cells, and $\mathrm{CK}-18^{+}$epithelial cells. Moreover, VSIG4 was coexpressed on $\mathrm{B} 7-\mathrm{H} 1^{+}$and $\mathrm{B} 7-\mathrm{H} 3^{+}$cells in these tumor specimens. Transfection of the VSIG4 gene into 293FT cells demonstrated that the VSIG4 signal could inhibit cocultured $\mathrm{CD}^{+}$and $\mathrm{CD}^{+}{ }^{+} \mathrm{T}_{\text {-cell }}$ proliferation and cytokine (IL-2 and IFN- $\gamma$ ) production in vitro. Interestingly, in a murine tumor model induced by Lewis lung carcinoma cell line, we found that tumors grown in VSIG4-deficient $\left(\mathrm{VSIG}^{-1-}\right)$ mice were significantly smaller than those found in wildtype littermates. All of these results demonstrate that macrophage-associated VSIG4 is an activator that facilitates lung carcinoma development. Specific targeting of VSIG4 may prove to be a novel, efficacious strategy for the treatment of this carcinoma.

Laboratory Investigation (2014) 94, 706-715; doi:10.1038/labinvest.2014.73; published online 26 May 2014

KEYWORDS: immunohistochemistry; lung carcinoma; macrophage; VSIG4

Lung cancer is the leading cause of cancer-related death worldwide. Patients with this carcinoma derive only limited benefits from standard surgical treatments, radiotherapy, chemotherapy, or adjuvant hormone therapies, and the 5year survival rate after diagnosis remains lower than $15 \%{ }^{1}$ Recently, multiple novel therapeutic strategies have been proposed. Most notably, approaches that aim to recruit the host's immune system to identify and destroy aberrant tumor cells have acquired considerable attention.

Effective immunotherapy against carcinoma depends on the presence of tumor-specific $\mathrm{CD} 8^{+}$cytotoxic T lymphocytes (CTLs). ${ }^{2}$ However, cancer cells often spread despite the relative abundance of CTLs in vivo. ${ }^{3}$ It has been suggested that complex or as yet undefined mechanisms may exist that prevent CTLs from eradicating metastatic cancer cells. The current double-signal model of cell-mediated interaction suggests that for optimal activation, $\mathrm{T}$ cells require both
TCR-mediated Ag-specific signals and a costimulatory signal provided by antigen presentation cells (APCs). Without sufficient costimulation, $\mathrm{T}$ cells are rendered anergic, tolerant, or apoptotic. ${ }^{4,5}$ The main costimulatory molecules are members of the B7 family such as B7.1 and B7.2, which stimulate T-cell activation by engaging with their receptor, $\mathrm{CD} 28$, on $\mathrm{T}$ cells. However, not all costimulatory molecules provide 'positive' signals; effector $\mathrm{T}$ cells also express several receptors that transfer 'negative' signals to terminate immune responses, such as the new CD28 family members CTLA-4, PD-1, and BTLA, ${ }^{6}$ which is termed as immunomodulatory molecules here. In addition, the newly defined $\mathrm{B} 7$ family members $\mathrm{PD}$ L1 (B7-H1), PD-L2 (B7-DC), B7-H3, and B7-H4 (B7x/B7-S1) are expressed on professional APCs as well as on cells within nonlymphoid organs, providing new means for downregulating T-cell activation and maintaining tolerance in peripheral tissues by serving as ligands of CD28. ${ }^{7}$ Disruption of negative

\footnotetext{
${ }^{1}$ Department of Oncology and Southwest Cancer Center, Southwest Hospital, Third Military Medical University, Chongqing, People's Republic of China and ${ }^{2}$ Institute of Immunology, PLA, Third Military Medical University, Chongqing, People's Republic of China

Correspondence: Professor Z Ruan, PhD, Department of Oncology and Southwest Cancer Center, Southwest Hospital, Third Military Medical University, 30 Gaotanyan Street, District Shapingba, Chongqing 400039, People's Republic of China.
}

E-mail: rzh1234@163.com

Received 5 September 2013; revised 17 April 2014; accepted 24 April 2014 
T-cell immunomodulatory pathways, using targeted disruption of gene expression, monoclonal antibodies, or soluble receptors that neutralize the coinhibitory signals, can overcome T-cell tolerance and generate effective antitumor responses. ${ }^{8,9}$

Coinhibitory signals have been implicated in the development of lung cancer. For instance, B7-H1 was focally observed in non-small-cell lung cancer (NSCLC) specimens and has been shown to inhibit T-cell infiltration into tumor tissues, indicating that $\mathrm{B} 7-\mathrm{H} 1$ may contribute to the negative regulation of antitumor immune responses to NSCLC. ${ }^{10}$ The expression of $\mathrm{B} 7-\mathrm{H} 3$ and $\mathrm{B} 7-\mathrm{H} 4$ was more common in cases with lymph node metastasis, suggesting their potential contribution to tumor progression in NSCLC patients. ${ }^{11}$ It would be interesting to investigate whether other members of the B7 superfamily are involved in this pathogenesis.

V-set and Ig domain-containing 4 (VSIG4, also called $\mathrm{CRIg}^{12}$ or $\mathrm{Z} 39 \mathrm{Ig}^{13}$ ) is a recently identified B7-related protein. ${ }^{14}$ VSIG4 is not only a receptor for complement C3, which mediates the clearance of pathogens, ${ }^{12}$ but also has the capacity to inhibit $\mathrm{CD} 4^{+}$and $\mathrm{CD} 8{ }^{+}$T-cell proliferation and IL-2 production by ligating an unidentified receptor on $\mathrm{T}$ cells. ${ }^{14}$ VSIG4 mRNA is highly expressed in various tissues, especially the liver, lung, placenta, and possibly the central nervous system, ${ }^{15-17}$ but its protein form is restricted to the surface of macrophages. ${ }^{12}$ Recent studies have shown that VSIG4 ${ }^{+}$cells are found in tissues from patients with rheumatoid arthritis, atherosclerosis (AS), and chronic HBV-infected livers, indicating that VSIG4 might be involved in the pathogenesis of these inflammatory diseases. ${ }^{13,18}$ However, the role of VSIG4 in tumor development is not clear.

This study investigated the characteristic expression of VSIG4 in patients with NSCLC with an immunostaining assay, and analyzed its in vivo role in tumor progression in a VSIG4-knockout mouse model. Our data indicated that VSIG4 facilitated tumor growth by inhibiting T-cell proliferation and cytokine production, which suggests that targeting VSIG4 might be a novel strategy for the treatment of this carcinoma.

\section{MATERIALS AND METHODS Patients}

Ten primary tumor specimens were obtained by surgery from patients with NSCLC (seven males, three females, mean age at diagnosis 50.1 years) who received no preoperative therapy. The samples were collected by the Department of Pathology, Southwest Hospital, Third Military Medical University, China, between 2006 and 2009. The tumors included four cases of adenocarcinomas and six cases of squamous cell carcinomas (two well-differentiated and eight poorly differentiated). The patients were staged (one case of stage I, six cases of stage II, and three cases of stage IIIA) according to their respective surgical and pathological findings based on the guidelines described in the American Joint
Committee on Cancer Staging Manual. ${ }^{19}$ Cell differentiation was determined using the current classification by the WHO, as revised in $1999 .{ }^{20}$ For all patients, surgical records, in-patient medical records, chest X-ray films, and whole-body computed tomography films were reviewed. This study was obtained with written informed consent from all participants involved, and was approved by the ethics committee of Southwest Hospital for Clinical Investigation.

\section{Mice}

VSIG4-deficient (VSIG4 ${ }^{-/-}$) C57BL/6 mice were provided by $\mathrm{Dr}$ van Lookeren Campagne $M$ (Department of Immunology, Genentech, South San Francisco, CA, USA). Age-matched male C57BL/6 wild-type (WT) control mice were purchased from the Animal Center of the Beijing University School of Medicine. All mice were maintained in microisolator cages and housed in the animal colony at the Animal Center, Third Military Medical University, and were fed a standard laboratory diet and water. All experiments were approved by and conducted in accordance with the guidelines of the Animal Care and Use Committee of the Third Military Medical University.

\section{Animal Studies and Tumor Models}

Murine Lewis lung carcinoma (LLC) cell lines were purchased from the Shanghai Institute of Biochemistry and Cell Biology, Chinese Academy of Sciences (Shanghai, China). ${ }^{21}$ Cells were cultured in complete medium containing RPMI1640 (Gibco, Uruguay), 10\% heat-inactivated fetal bovine serum (Hyclone Laboratories, Logan, UT, USA), penicillin and streptomycin $(100 \mu \mathrm{g} / \mathrm{ml})$, L-glutamine $(2 \mathrm{mM})$, and HEPES $(10 \mathrm{mM})$.

A total of $2 \times 10^{5}$ LLC tumor cells were subcutaneously injected into 11 -week-old male VSIG4 $4^{-1-}$ mice as well as their WT littermates. The long (L) and short (S) axes of the tumor were measured with calipers every 3 days, and tumor volume was calculated using the formula $L \times S^{2} \times 0.5$, as described by Euhus et al. ${ }^{22}$

\section{Immunohistochemical and Immunofluorescent Staining}

Fresh lung carcinoma specimens of patients were embedded in OCT compound (Tissue Tek, Tokyo, Japan) and stored at $-80^{\circ} \mathrm{C}$ until use. Seven-micrometer cryostat sections were prepared for staining. Briefly, the sections were fixed in acetone $\left(-20^{\circ} \mathrm{C}, 5 \mathrm{~min}\right)$, air-dried, and blocked with $1 \%$ BSA in PBS. The specimens were then incubated with mouse anti-human VSIG4 (provided by Dr Campagne, Genentech, USA, and Dr Won-Ha Lee, Department of Genetic Engineering, Kyungpook National University, Taegu, Korea) for $8 \mathrm{~h}$ at $4{ }^{\circ} \mathrm{C}$. The sections were washed and incubated with a secondary polymeric, peroxidase-labeled rabbit anti-mouse antibody (EnVision ${ }^{\mathrm{TM}}$ System, Dako, Copenhagen, Denmark) for $30 \mathrm{~min}$. Negative controls were processed by substituting an isotype-matched, concentration-matched immunoglobulin to exogenous targets for the primary antibody. 
Reactivity was detected with a DAB Elite Kit (K3465; Dako, Copenhagen, Denmark) and brown coloration of tissues indicated positive staining. To determine which cell types express VSIG4, immunofluorescent double staining was performed. The sections were incubated overnight with antiVSIG4, anti-CD68 (rabbit IgG, 1:100; Santa Cruz, San Diego, CA, USA), CK-18 (mouse rabbit IgG, 1:50; Santa Cruz), CD31 (rabbit IgG, 1:50; Santa Cruz), and CD3 antibodies (mouse IgG, 1:50; Dako), followed by fluorescenceconjugated secondary antibody (FITC- or CY3-labeled antimouse or anti-rabbit IgG 1:100; Zymed, San Diego, CA, USA). However, sections incubated with the related isotype control antibodies with added fluorescence-labeled second antibodies were used as isotype controls. The nuclei were stained with DAPI. Results were analyzed by fluorescence microscopy (Zeiss Axioplan 2, Berlin, Germany).

The fresh murine LLC specimens were prepared with the same protocol of patients' lung carcinoma specimens. The anti-mouse VSIG4 (goat IgG, 1:50; Santa Cruz) was used to detect the macrophage in the specimens by using immunofluorescent staining which was described above.

\section{Human VSIG4 (Z39lg) Clone and Vector Construction}

Human monocytes from PBMCs were purified and isolated, and total RNA was purified using Trizol (Invitrogen, Carlsbad, CA, USA) as described previously. The human VSIG4 gene was amplified by RT-PCR. The sense primer $\left(5^{\prime}\right.$-GTGTC CAGTTTGGCTAGTGCC- $\left.3^{\prime}\right)$ and antisense primer $\left(5^{\prime}\right.$-GACT GGAGAACAGAAGCAGGC-3') were designed for the cloning of a $1.2 \mathrm{~kb}$ fragment of the whole human VSIG4 gene. The cloned fragment was inserted into an FUGW plasmid with $B g l I I$ and BsrGI sites to generate FUGW-VSIG4, and the sequence of VSIG4 oligonucleotides was verified by DNA sequencing (Invitrogen).

\section{Recombinant Lentivirus Preparation}

Recombinant Lentivirus was prepared as described in Tronolab (http://tronolab.epfl.ch). Briefly, the lentiviral envelope plasmid (psPAX2, $4.5 \mu \mathrm{g}$ ), the packaging plasmid (pMD2G, $1.8 \mu \mathrm{g}$ ), and the expression plasmids (FUGW or FUGWVSIG4, $6 \mu \mathrm{g}$, respectively) were added and mixed in $703.5 \mu \mathrm{l}$ distilled water plus $46.5 \mu \mathrm{l} \mathrm{CaCl}_{2}(2 \mathrm{~mol} / \mathrm{l})$.

The 293FT cells, which were also purchased from the Shanghai Institute of Biochemistry and Cell Biology, were further treated with the recombinant lentivirus. Briefly, $4 \times 10^{6} 293 \mathrm{FT}$ cells were plated in a 10 -cm cell culture dish. At $80-90 \%$ confluence, cells were treated with chloroquine ( $3 \mu \mathrm{l} 25 \mathrm{mM}$ per well) for $1 \mathrm{~h}$ and lentivirus was added. After $48 \mathrm{~h}$, the supernatant was collected and stored at $4{ }^{\circ} \mathrm{C}$. The supernatant was collected and centrifuged at $23000 \mathrm{~g}$ for $6 \mathrm{~h}$ to concentrate the virus. To further purify the product, the viral preparation was laid on $20 \%$ sucrose and centrifuged at $50000 \mathrm{~g}$ for $2 \mathrm{~h}$. The resultant virus pellets were resuspended in HBSS.

\section{Cell Transfection}

In total, $4 \times 10^{5}$ per well 293FT cells were cultured in 6-well culture plates to $60-70 \%$ confluence. Cells were treated with the lentivirus ( $1 \mathrm{ml}$ per well) and washed with DMEM after $7 \mathrm{~h}$. The transfected 293FT cells were further cultured for $48 \mathrm{~h}$ and VSIG4 expression was detected by flow cytometry (FACsAria Cytometer; Becton Dickinson, Berlin, Germany). Briefly, suspended cells were incubated for $30 \mathrm{~min}$ at room temperature in the dark using anti-VSIG4 primary antibodies. After $30 \mathrm{~min}$, FITC-anti-mouse IgG1 secondary antibodies (eBioscience, San Diego, CA, USA) were added, and the cells were incubated for an additional $1 \mathrm{~h}$. After a subsequent PBS wash, 10000 cells were acquired. Flow cytometric data were analyzed using the CellQuest Pro software. The 293FT cells with significant VSIG4 expression were named 293FT-VSIG4, and cells transfected with the control vector were labeled 293FT-FU.

\section{Coculture Experiments}

$\mathrm{CD}^{+}$and $\mathrm{CD}^{+}{ }^{+} \mathrm{T}$ cells were isolated from the PBMCs of normal individuals. We also obtained written informed consent from all participants as well as the approval of the ethics committee of South-West Hospital for Clinical Investigation. Cells were isolated by negative or positive selection using a $\mathrm{CD}^{+}{ }^{+} \mathrm{T}$-cell Isolation Kit II or $\mathrm{CD} 8$ Microbeads, respectively (Miltenyi Biotech, Auburn, CA, USA). T cells were cocultured with 293FT-VSIG4 cells or control 293FT-FUGW cells in the presence of anti-CD3 mAbs ( $10 \mathrm{ng} / \mathrm{ml}$, clone HIT3a; eBioscience) for a total of $72 \mathrm{~h}$. T-cell proliferation was measured by MTT, and IL- 2 and IFN- $\gamma$ production in the cocultured supernatant was detected by ELISA (see below).

\section{MTT Assay}

Primary $\mathrm{CD}^{+}$and $\mathrm{CD} 88^{+} \mathrm{T}$ cells were cocultured with $293-$ VSIG4 cells in the presence of anti-CD3 $\mathrm{mAbs}(10 \mathrm{ng} / \mathrm{ml})$. $\mathrm{T}$ cells were collected after $72 \mathrm{~h}$, and cell proliferation was measured by MTT according to the manufacturer's instructions (Invitrogen). Briefly, collected $\mathrm{T}$ cells were washed with warm RPMI-1640 without phenol red. Then, MTT working solution was added into the assay wells and incubated at $37^{\circ} \mathrm{C}$ for $2 \mathrm{~h}$. The converted dye was solubilized with $1 \mathrm{ml}$ of acidic isopropanol $(0.04 \mathrm{M} \mathrm{HCl}$ in absolute isopropanol). The dye solution containing the cells was transferred into 1.5-ml Eppendorf tubes and centrifuged at 13000 r.p.m. for $2 \mathrm{~min}$, and the supernatant was transferred into a second Eppendorf tube. Absorbance of the converted dye was measured at a wavelength of $570 \mathrm{~nm}$ with background subtraction at $650 \mathrm{~nm}$. A Beckman DU-600 spectrophotometer and disposable plastic cuvettes were used for measurements.

\section{ELISA}

Levels of IL-2 and IFN- $\gamma$ in the cocultured supernatant were detected using an IL-2 or IFN- $\gamma$ Enzyme-Linked 
Immunosorbent Assay Kit, according to the manufacturer's instructions (eBioscience).

\section{Statistical Analysis}

Differences in tumor volume between VSIG4 ${ }^{-1-}$ and WT mice were analyzed statistically with Student's $t$-test. Mouse survival rates were analyzed using the log-rank test. Results where $P<0.05$ were considered to be statistically significant.

\section{RESULTS}

\section{The Characteristic Expression of VSIG4 Protein in Human Lung Carcinoma Tissues}

Carcinoma tissues and pericarcinoma tissues from patients with NSCLC were collected and stained with hematoxylin and eosin (H\&E) (Figures 1a and d), followed by immunohistochemistry for the expression of VSIG4. The results showed that VSIG4 protein was observed in all of the tissues analyzed, and $\mathrm{VSIG}^{+}$cells were distributed throughout every NSCLC sample (Figures $1 \mathrm{~b}$ and $\mathrm{c}$ ). From the pericarcinoma tissues, we found that the VSIG4 ${ }^{+}$cells were distributed in the lung interstitium and alveolar cavity, but absent from the endothelial cells of blood vessels (Figures 1e and f). However, the isotype antibody control did not show any positive signals (Figure 1g). Results also indicated that VSIG4 $^{+}$cells were infiltrated macrophages, rather than carcinoma cells. At the cellular level, VSIG4 expression was restricted in the cytoplasm and on the cell surface, but it was absent from the nuclei (Figure 1).

Immunofluorescent double staining showed that VSIG4 was expressed on $\mathrm{CD}_{68}{ }^{+}$macrophages, but it was not expressed on infiltrated $\mathrm{CD}^{+} \mathrm{T}$ cells, $\mathrm{CD} 31^{+}$vascular endothelial cells, or CK-18 ${ }^{+}$epithelial cells in samples from NSCLC patients (Figure 2). High levels of VSIG4 were found on tumor-infiltrated macrophages, suggesting that this molecule might be involved in the pathogenesis of NSCLC.

The expression of costimulatory molecules of the $\mathrm{B} 7$ superfamily, including $\mathrm{B} 7-\mathrm{H} 1, \mathrm{~B} 7-\mathrm{DC}, \mathrm{B} 7-\mathrm{H} 3$, and $\mathrm{B} 7-\mathrm{H} 4$, is enhanced in NSCLC patients. ${ }^{10,11}$ We further detected the expression pattern of these molecules in cancer tissue from NSCLC patients in this study by immunohistochemistry. Results showed that all B7-H1, B7-DC, B7-H3, and B7-H4 molecules were expressed on cancer cells (Figures 3a, c, e, and g).
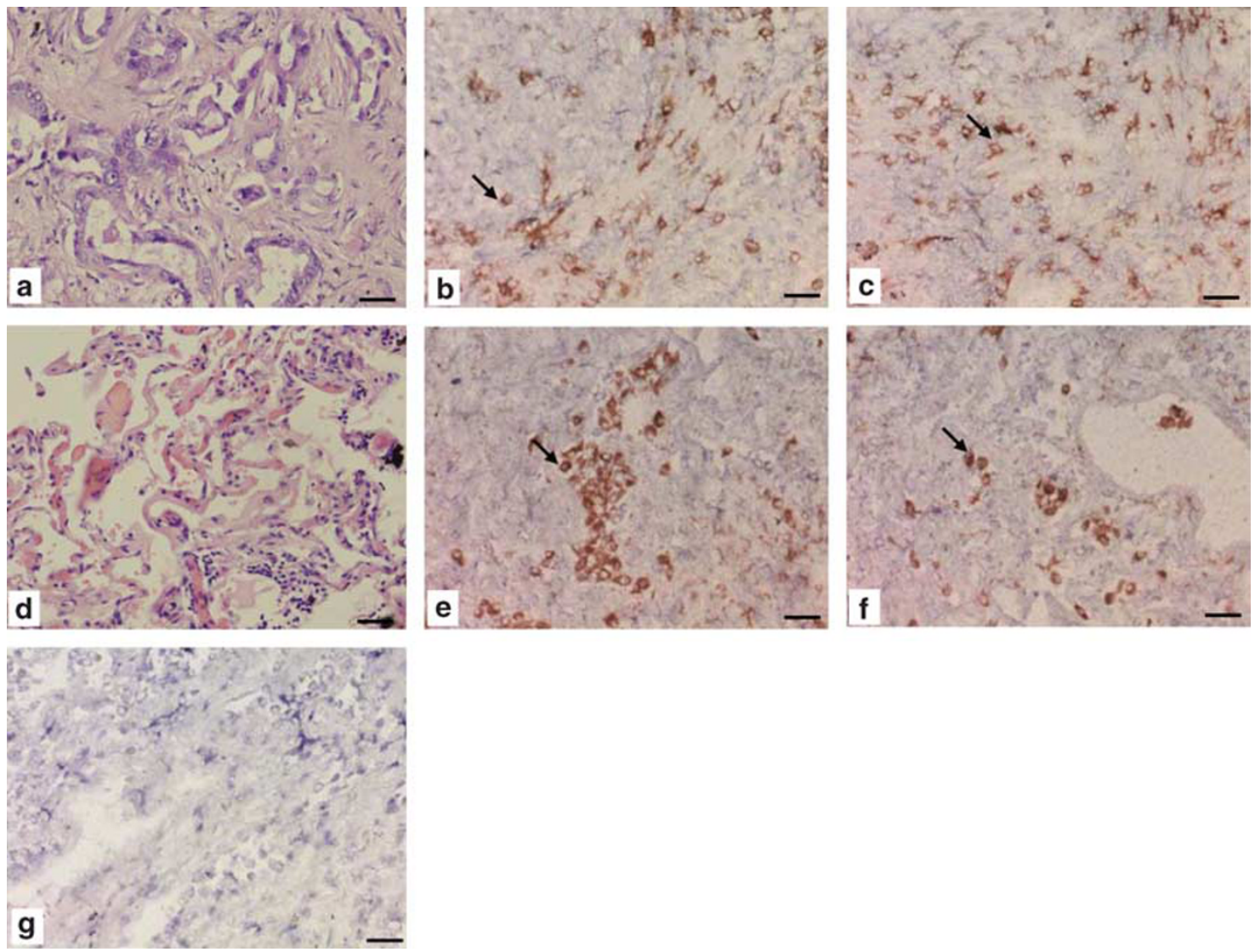

Figure 1 The characteristic expression of V-set and Ig domain-containing 4 (VSIG4) in malignant sections from non-small-cell lung cancer (NSCLC) patients detected by immunohistochemistry. Seven-micrometer cryostat sections of carcinoma tissues and pericarcinoma tissues from patients with NSCLC were prepared and stained with hematoxylin and eosin (H\&E) (a and $\mathbf{d})$. The sections of carcinoma tissues (b and $\mathbf{c})$ and pericarcinoma tissues (e and f) were then detected for the expression of VSIG4 by immunohistochemistry with the mouse anti-human VSIG4 as the detecting antibody. The isotype antibody was set as control for the immunohistochemistry assay (g). Reactivity was detected with a DAB Elite kit. The arrow indicates positive cells. Scale bar $=20 \mu \mathrm{m}$. 

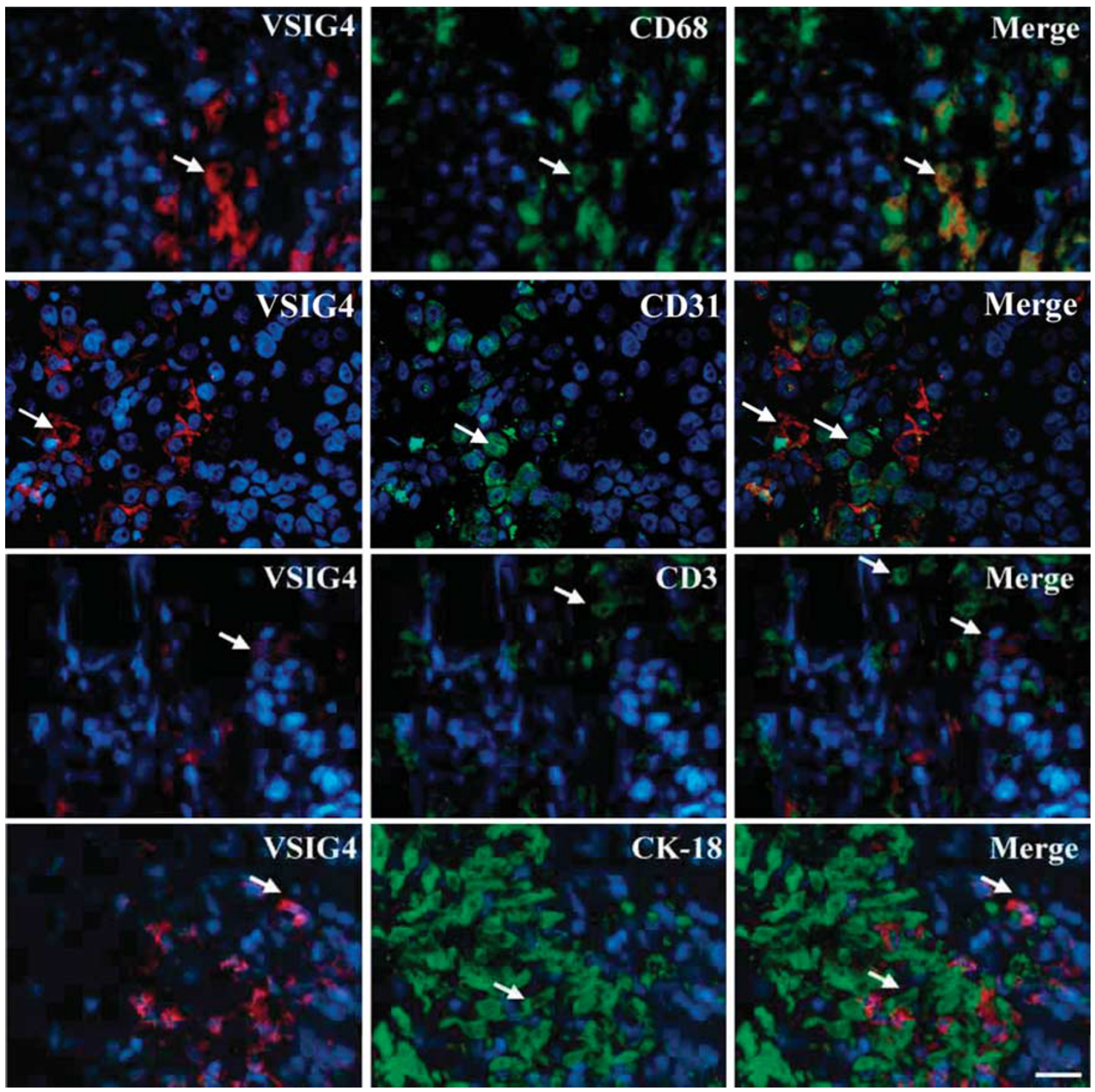

Figure 2 The characteristic expression of V-set and Ig domain-containing 4 (VSIG4) in malignant sections from non-small-cell lung cancer (NSCLC) patients detected with immunofluorescent staining. Sections of lung cancer tissues were incubated with anti-VSIG4, anti-CD68, anti-CK-18, anti-CD31, and anti-CD3 antibodies, followed by incubation with fluorescence-conjugated secondary antibodies and analysis by fluorescence microscopy. Arrows indicate positive cells; blue color indicates nuclear 4',6-diamidino-2-phenylindole (DAPI) staining. Scale bar $=20 \mu \mathrm{m}$.

While B7-H1, B7-H3, and B7-H4 were observed on infiltrated inflammatory cells (Figures $3 \mathrm{~b}$ and d), B7-H3 was also found on capillaries (Figure 3f). We further analyzed whether VSIG4 was coexpressed with these molecules by immunofluorescent dual staining. Results showed that the expression of VSIG4 was found on $\mathrm{B} 7-\mathrm{H}^{+}{ }^{+}$and $\mathrm{B} 7-\mathrm{H}_{3}{ }^{+}$ cells, but it was absent from $\mathrm{B} 7-\mathrm{DC}^{+}$and $\mathrm{B} 7-\mathrm{H} 4^{+}$cells (Figure 4).

\section{Functional Relevance of Macrophage-Associated VSIG4: Inhibition of Cocultured T-Cell Activation}

To describe the exact function of VSIG4 in T-cell activation, the human VSIG4 gene was cloned and then transfected into
293FT cells. Flow cytometry indicated that the majority of VSIG4 plasmid-transfected cells expressed VSIG4 (Figure 5a). The 293FT-VSIG4 and control (293FT-FUGW) cells were cocultured with primary human $\mathrm{CD} 4^{+}$and $\mathrm{CD} 8^{+} \mathrm{T}$ cells. The cells were collected, and cell proliferation was measured by MTT assay after 3 days. The results showed that the proliferation of both $\mathrm{CD}^{+}$and $\mathrm{CD}^{+}{ }^{+} \mathrm{T}$ cells was significantly inhibited by the VSIG4 signal (Figure 5b). In parallel, the secretion of IL-2 and IFN- $\gamma$ into the supernatant was also suppressed compared with that of $\mathrm{T}$ cells cocultured with 293FT-FUGW cells (Figure 5c). These results demonstrate that VSIG4 can inhibit cocultured T-cell proliferation and cytokine production. 

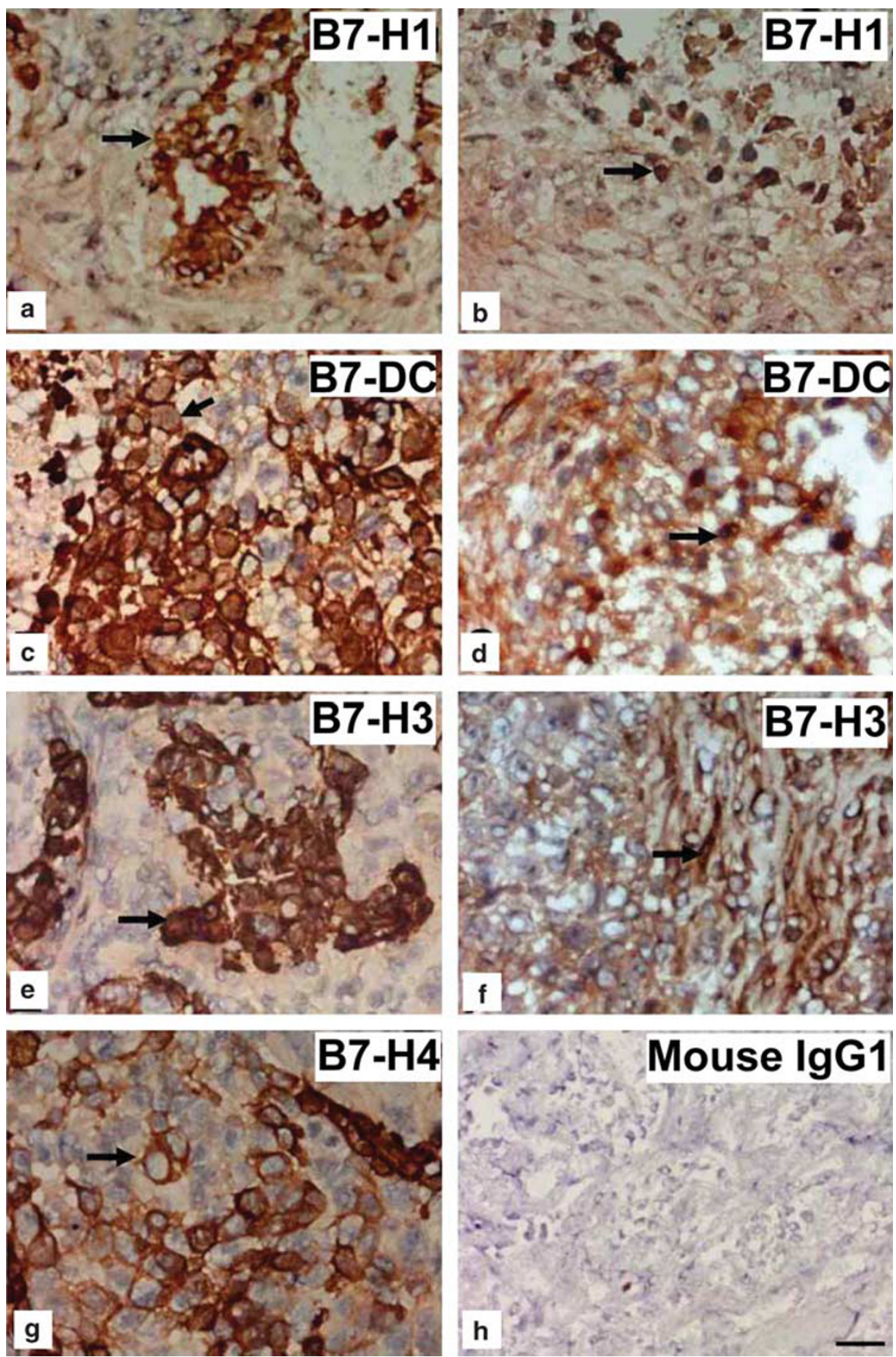

Figure 3 Expression of B7-H1, B7-DC, B7-H3, and B7-H4 in malignant tissue from non-small-cell lung cancer (NSCLC) patients detected by immunohistochemistry. Expression of B7-H1 (a and b), B7-DC (c and d), B7-H3 (e and $\mathbf{f}$ ), and B7-H4 (g) on cancer cells (left column) and infiltrated inflammatory cells (right column) was detected by immunohistochemistry with the indicated monoclonal antibodies (mAbs), respectively. (h) Mouse immunoglobulin $\mathrm{G}(\mathrm{IgG})$ isotype control. The arrow indicates positive cells. Scale bar $=20 \mu \mathrm{m}$.

\section{Tumor Growth was Suppressed Significantly in VSIG4-Deficient Mice}

To determine the effect of macrophage-associated VSIG4 on the growth of lung carcinoma in vivo, LLC cell lines were injected subcutaneously into VSIG4 ${ }^{-1-}$ mice or WT littermates, and the tumor volume was measured over the subsequent 4 weeks. The results showed that tumor volume in VSIG4 ${ }^{-1-}$ mice was markedly smaller than that of WT littermates at 38 days after inoculation (Figure 6a). Immunofluorescence assay showed that VSIG4 ${ }^{+}$macrophages infiltrated into the tumor tissue in WT mice; however, no VSIG $^{+}$macrophages could be observed in tumor tissues of 

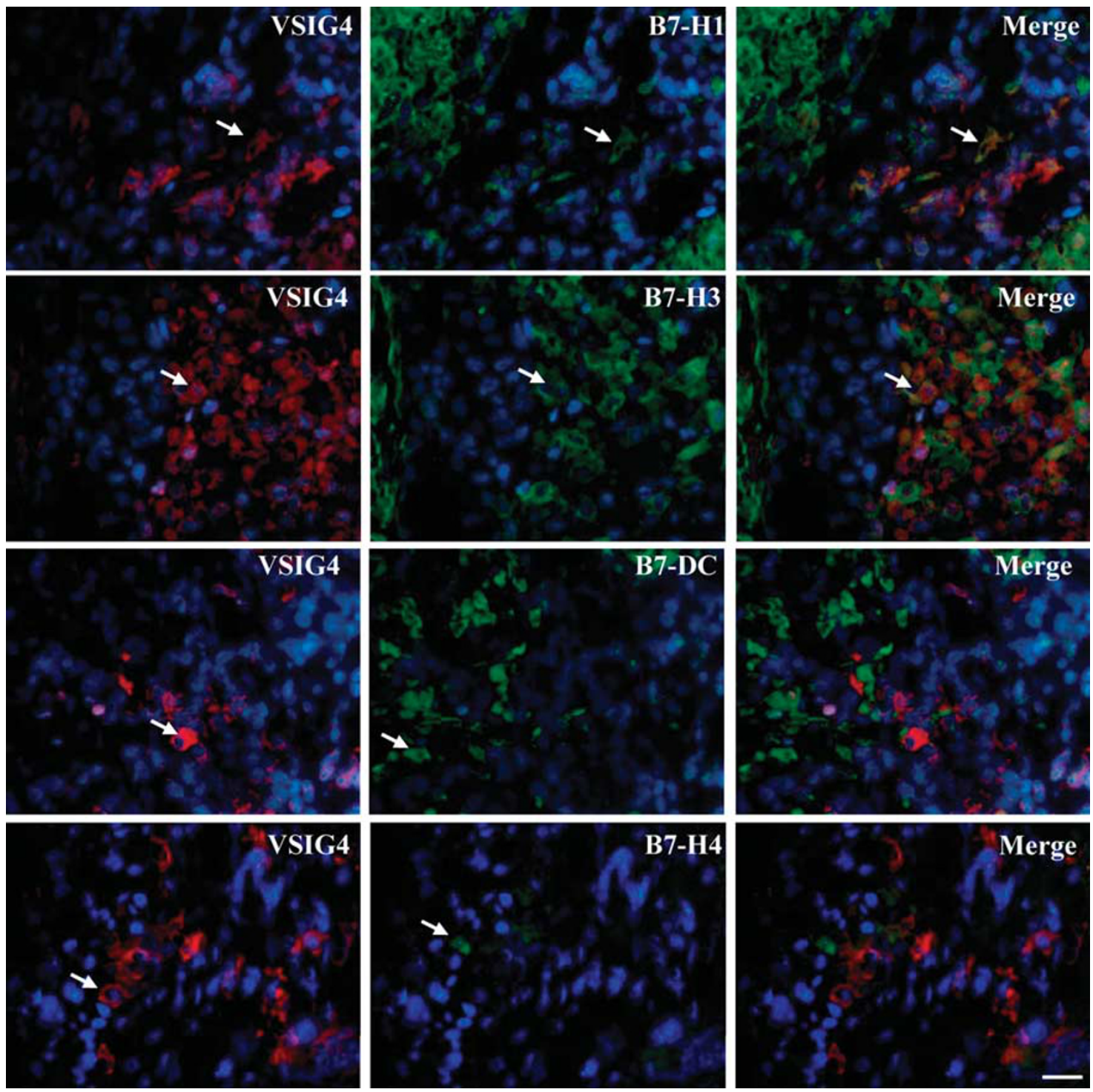

Figure 4 Coexpression of V-set and Ig domain-containing 4 (VSIG4) with B7-H1, B7-DC, B7-H3, or B7-H4 in malignant tissue from non-small-cell lung cancer (NSCLC) patients detected with immunofluorescent double staining. Sections of lung cancer tissues were incubated with anti-VSIG4, anti-B7-H1, anti-B7-DC, anti-B7-H3, and anti-B7-H4 antibodies, followed by incubation with fluorescence-conjugated secondary antibodies and analysis by fluorescence microscopy. The arrow indicates positive cells; nuclei were stained with 4',6-diamidino-2-phenylindole (DAPI). Scale bar $=20 \mu \mathrm{m}$.

$\mathrm{VSIG}^{-1-}$ mice (Figure $\left.6 \mathrm{~b}\right)$. These results collectively indicate that VSIG4 facilitates the growth of LLC cells in vivo.

\section{DISCUSSION}

Research in tumor immunology has provided a wealth of information about tumor evasion strategies. Despite this knowledge, the etiology and pathogenesis of carcinomas remain unclear. Depressed immune responses are frequently observed in cancer patients, suggesting the possible existence of as yet unspecified or perhaps complex immunosuppressive factors that affect the antitumor capacity of CTLs. Current research has demonstrated that malignant lung cancer cells inhibit the immune responses of CTLs, possibly by the release of suppressive factors (eg, IL-6, TGF- $\beta,{ }^{23}$ or IL-10 (ref. 24)), the expression of death ligand CD95L, ${ }^{23}$ the absence of costimulatory molecules (B7.1 and B7.2), an increase in the expression of immunomodulatory molecules (B7-H1, B7-DC, B7-H3, and B7-H4), or an enhancement of the pool of CD $4{ }^{+} \mathrm{CD} 25^{+}$regulatory T cells. ${ }^{25}$ All of these factors can induce CTL anergy or tolerance, thus preventing tumor destruction.

Tumor stromata are required for the survival, growth, and progression of cancers. The major components in tumor stromata are fibroblasts and tumor-associated macrophages. Stromata have the capacity to inhibit tumor T-cell infiltration and activation. ${ }^{26,27}$ However, the mechanisms by which 

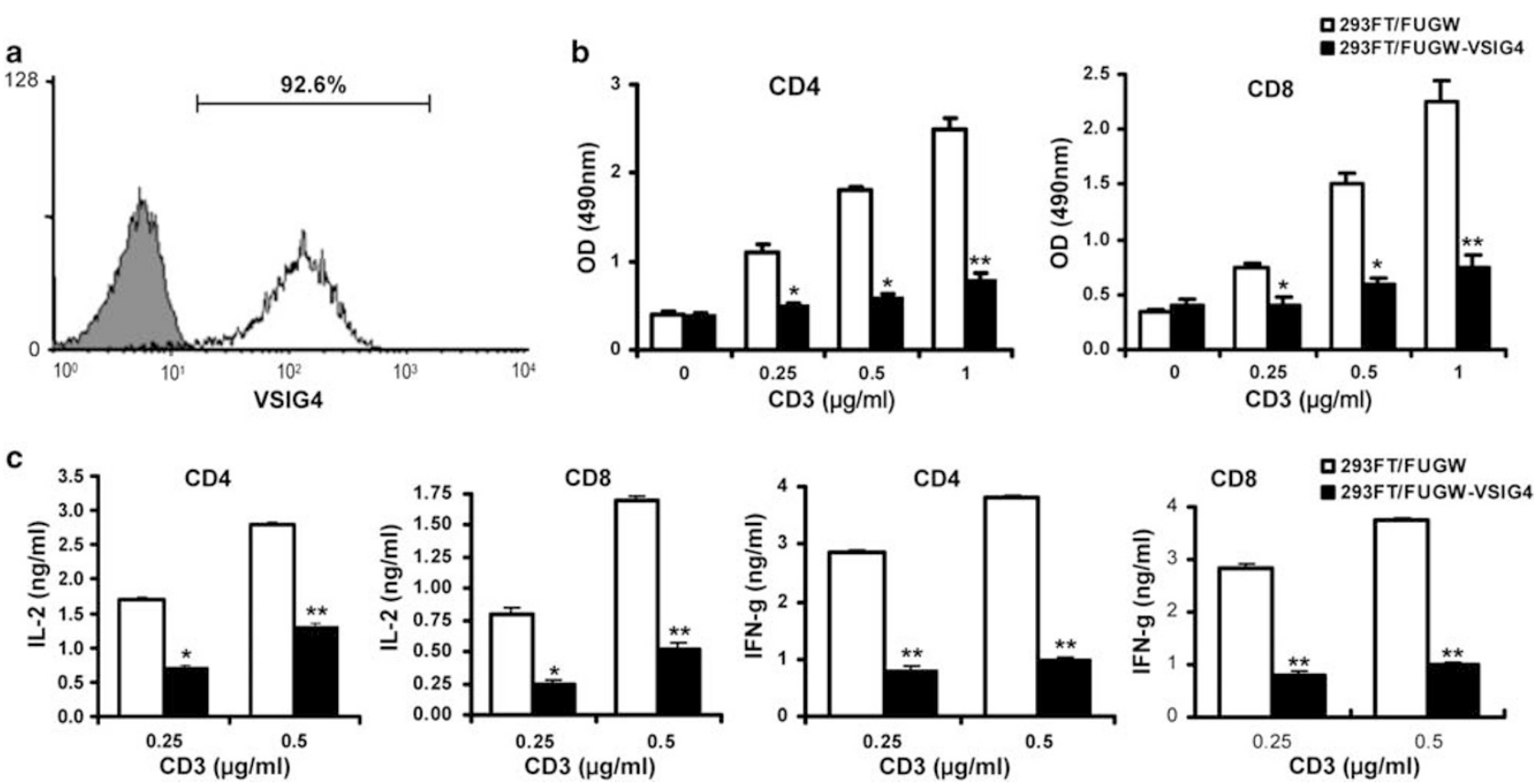

Figure 5 V-set and Ig domain-containing 4 (VSIG4) inhibits cocultured T-cell proliferation and cytokine production. (a) The 293FT cells were transfected with lentivirus-expressing VSIG4 or empty lentivirus (gray shadow), and VSIG4 expression was confirmed by fluorescence-activated cell sorter (FACS). (b) Purified CD4 ${ }^{+}$and $\mathrm{CD}^{+}{ }^{+}$T cells were cocultured with 293FT or 293FT-VSIG4 cells, and cell proliferation was measured by 3-[4,5-dimethylthiazol-2-yl]2,5 diphenyl tetrazolium bromide (MTT) after 3 days. (c) Interleukin-2 (IL-2) and interferon- $\gamma$ (IFN- $\gamma$ ) concentrations in the cocultured supernatant were measured by enzyme-linked immunosorbent assay (ELISA). Values represent the mean of triplicate wells of at least three independent experiments and the data were shown by mean \pm s.e.m. ${ }^{*} P<0.05 ;{ }^{*} P<0.001$.
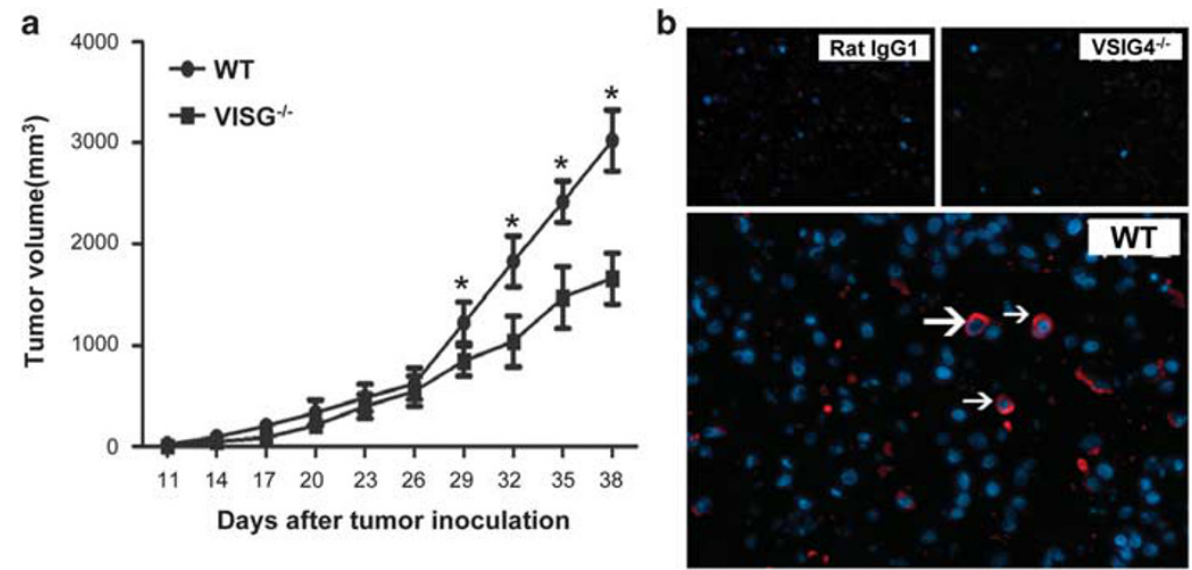

Figure 6 The development of Lewis lung carcinoma was inhibited in VSIG4 ${ }^{-1-}$ mice. A total of $2 \times 10^{5}$ LLC tumor cells were subcutaneously injected into the lower right of 11 -week-old male VSIG4 ${ }^{-1-}$ mice or sex- and age-matched C57BL/6 WT mice. (a) Tumor growth was observed every 3 days in WT and VSIG $4^{-1-}$ mice from 38 days after inoculation. Data were from three independent experiments and were shown as mean \pm s.e.m. Six mice per each group for each of three experiments were used. ${ }^{*} P<0.05$ vs VISG ${ }^{-1-}$ group. (b) The expression of VSIG4 in tumor tissue from VSIG4 ${ }^{-/-}$and WT mice was detected by immunofluorescent staining. Arrow indicates VSIG4-positive cells. VSIG4, V-set and Ig domain-containing 4.

tumor stromata contribute to tumor immunopathogenesis are poorly understood. ${ }^{28}$ Tumor macrophages represent an abundant population of APCs in solid tumors and are thought to subvert tumor-specific immunity. However, their suppressive roles and mechanisms are not well understood in the immunopathogenesis of cancers. ${ }^{29}$
VSIG4 is a recently identified immunomodulatory molecule of the B7 family. Constitutive expression of the VSIG4 protein is normally restricted in macrophage-lineage cells. The expression of VSIG4 in tumor tissues has not been reported, although its expression was observed in tissues from patients with RA, AS, or chronic HBV infection. ${ }^{13,18}$ 
In this study, we investigated the expression of VSIG4 in the tissue of 10 patients with NSCLC. Immunohistochemical staining revealed that VSIG4 ${ }^{+}$cells were observed in lung cancer tissue, and VSIG4 expression was restricted to the membranes and the cytoplasms of those cells. Dual fluorescent staining showed that VSIG4 was restricted to macrophages, whereas other cell types, including tumor cells, endothelial cells, and T cells, displayed no VSIG4 expression.

It has been shown that VSIG4 has the capacity to inhibit $\mathrm{CD}^{+}{ }^{+}$and $\mathrm{CD}^{+}{ }^{+}$T-cell proliferation and IL-2 production by ligating an unidentified receptor on T cells. ${ }^{14}$ Therefore, VSIG4 may be a potent negative regulator of T-cell responses. To further describe the exact function of VSIG4 in regulating immune response to tumor cells, we cloned the human VSIG4 gene into the pKE2 plasmid. The 293FT cells were transfected with this plasmid, and stably expressed VSIG4 clones were selected. The 293FT/T $\left(\mathrm{CD} 4^{+}\right.$and $\left.\mathrm{CD} 8^{+}\right)$cells were cocultured, and T-cell proliferation was markedly inhibited in the presence of VSIG4. Moreover, supernatants were harvested to detect cytokine release from $\mathrm{T}$ cells by ELISA after 3 days. After coculture with 293FT-VSIG4 cells, IL- 2 and IFN- $\gamma$ cytokine production levels were significantly lower than those observed in the 293FT-FUGW group or the untreated group. Therefore, VSIG4 has the capacity to inhibit cytokine release from cocultured $\mathrm{CD} 4^{+}$and $\mathrm{CD} 8{ }^{+} \mathrm{T}$ cells, and suppress T-cell proliferation. We thus propose that it is possible that tumors use this effect to escape recognition by the host's immune system. To further analyze macrophageassociated VSIG4 in tumor immunity, VSIG4 ${ }^{-1-}$ and WT mice were used to establish an LLC model. Both tumor growth and tumor volume in the VSIG4 ${ }^{-1-}$ mice were significantly lower than that in the WT litter since 29 days after tumor cell inoculation. This delayed tumor-inhibiting effects in VSIG4 ${ }^{-1-}$ mice might reflect the fact that there are other immune-inhibiting molecules in vivo besides VSIG4. ${ }^{6,7}$ The deficiency of VSIG4 might remove partial inhibition effects to host immune responses; however, other inhibitory molecules would partially compensate the function of VSIG4, which would cause the significant but delayed inhibition of tumor growth in VSIG4 ${ }^{-1-}$ mice.

Interestingly, in this study, we also found that VSIG4 was coexpressed with $\mathrm{B} 7-\mathrm{H}_{1}{ }^{+}$and $\mathrm{B} 7-\mathrm{H}_{3}{ }^{+}$cells within the tumor stroma. B7-H1, which normally expressed restrictedly in immune cells, has been shown that it can inhibit immune responses by inducing T-cell apoptosis, impairing cytokine production, and diminishing the cytotoxicity of activated $\mathrm{T}$ cells and may endow tumors with a mechanism to escape host immune destruction. ${ }^{7}$ B7-H3 has also been shown to inhibit T-cell responses by impaired T-cell stimulatory function. ${ }^{30,31}$ Thus, it probably means that both the VSIG4 signaling pathway and the B7-H1/B7-H3 signaling pathway synergistically contribute to the immunosuppressive effects of the $\mathrm{VSIG}^{+}$macrophages.

It has been well accepted that disruption of negative T-cell immunomodulatory pathways using targeted disruption strategies such as knocking down gene expression, and applying monoclonal antibodies or soluble receptors that neutralize the coinhibitory signals, can overcome T-cell tolerance and generate effective antitumor responses. ${ }^{8,9}$ The suppressive function of VSIG4 as determined in this study might have some important implications for the immunobiology of lung cancer, and targeting the VSIG4 pathway in combination with a vaccination using the recently identified CTL epitope TRAG-3 $3_{58-66}$ (ref. 32) might aid in the design of a novel regimen for specific tumor immunotherapy. In this case, while the suppressive effects of VSIG- $4^{+}$macrophages were inhibited, the TRAG-specific antitumor immune response will be amplified.

Owing to the facility of infection with the recombinant lentivirus, the 293FT cells were used to express VSIG4 gene by transfection, and the transfected 293FT cells did demonstrate the significant immunosuppressive effects. However, the 293FT cells might have different intrinsic features to macrophages. Therefore, it will be more helpful to force the expression of VSIG4 gene in a macrophage cell line lacking VSIG4 gene, or knock down the expression of VSIG4 gene in a VSIG4 ${ }^{+}$macrophage cell line to investigate the natural function of VSIG4 gene in the future.

\section{ACKNOWLEDGMENTS}

We thank Dr van Lookeren Campagne M (Department of Immunology, Genentech, South San Francisco, CA, USA) and Dr Won-Ha Lee (Department of Genetic Engineering, Kyungpook National University, Taegu, Korea) for human VSIG4 mAbs. We also thank Dr Changsong Wang and Jianjun Liu for their photographic assistance and their insightful pathological observations. This work is supported by The Natural Science Foundation of China (30872374, 30490241 and 30971099).

\section{DISCLOSURE/CONFLICT OF INTEREST}

The authors declare no conflict of interest.

1. Bach PB, Mirkin JN, Oliver TK, et al. Benefits and harms of CT screening for lung cancer: a systematic review. JAMA 2012;307:2418-2429.

2. Boon T, Cerottini JC, Van den Eynde B, et al. Tumor antigens recognized by T lymphocytes. Annu Rev Immunol 1994;12:337-365.

3. Wilcox RA, Flies DB, Zhu G, et al. Provision of antigen and CD137 signaling breaks immunological ignorance, promoting regression of poorly immunogenic tumors. J Clin Invest 2002;109:651-659.

4. Baxter AG, Hodgkin PD. Activation rules: the two-signal theories of immune activation. Nat Rev Immunol 2002;2:439-446.

5. Schwartz RH. A cell culture model for T lymphocyte clonal anergy. Science 1990;248:1349-1356.

6. Chen L. Co-inhibitory molecules of the B7-CD28 family in the control of T-cell immunity. Nat Rev Immunol 2004;4:336-347.

7. Wang YH, Cao YW, Yang XC, et al. Effect of TLR4 and B7-H1 on immune escape of urothelial bladder cancer and its clinical significance. Asian Pac J Cancer Prev 2014;15:1321-1326.

8. Nishimura H, Okazaki T, Tanaka Y, et al. Autoimmune dilated cardiomyopathy in PD-1 receptor-deficient mice. Science 2001;291: 319-322.

9. Egen JG, Kuhns MS, Allison JP. CTLA-4: new insights into its biological function and use in tumor immunotherapy. Nat Immunol 2002;3: 611-618.

10. Konishi J, Yamazaki K, Azuma M, et al. B7-H1 expression on non-small cell lung cancer cells and its relationship with tumor-infiltrating lymphocytes and their PD-1 expression. Clin Cancer Res 2004;10: 5094-5100. 
11. Sun $\mathrm{Y}$, Wang $\mathrm{Y}$, Zhao J, et al. B7-H3 and B7-H4 expression in non-smallcell lung cancer. Lung Cancer 2006;53:143-151.

12. Helmy KY, Katschke Jr KJ, Gorgani NN, et al. CRlg: a macrophage complement receptor required for phagocytosis of circulating pathogens. Cell 2006;124:915-927.

13. Lee MY, Kim WJ, Kang YJ, et al. Z39lg is expressed on macrophages and may mediate inflammatory reactions in arthritis and atherosclerosis. J Leukoc Biol 2006;80:922-928.

14. Vogt L, Schmitz N, Kurrer MO, et al. VSIG4, a B7 family-related protein, is a negative regulator of T cell activation. J Clin Invest 2006; 116:2817-2826.

15. Walker MG. Z39lg is co-expressed with activated macrophage genes. Biochim Biophys Acta 2002;1574:387-390.

16. Kim JK, Choi EM, Shin $\mathrm{HI}$, et al. Characterization of monoclonal antibody specific to the Z39lg protein, a member of immunoglobulin superfamily. Immunol Lett 2005;99:153-161.

17. Kammula EC, Motter J, Gorgels A, et al. Brain transcriptome-wide screen for HIV-1 Nef protein interaction partners reveals various membrane-associated proteins. PLoS One 2012;7:12.

18. Guo S, Yang C, Mei F, et al. Down-regulation of Z39lg on macrophages by IFN-gamma in patients with chronic HBV infection. Clin Immunol 2010;136:282-291.

19. Fleming ID, Phillips JL, Menck HR, et al. The National Cancer Data Base report on recent hospital cancer program progress toward complete American Joint Committee on Cancer/TNM staging. Cancer 1997; 80:2305-2310

20. Hensing TA. Clinical evaluation and staging of patients who have lung cancer. Hematol Oncol Clin N Am 2005;19:219-235, v.

21. Zhou HJ, Zhang JL, Li A, et al. Dihydroartemisinin improves the efficiency of chemotherapeutics in lung carcinomas in vivo and inhibits murine Lewis lung carcinoma cell line growth in vitro. Cancer Chemother Pharm 2010;66:21-29.
22. Euhus DM, Hudd C, LaRegina MC, et al. Tumor measurement in the nude mouse. J Surg Oncol 1986;31:229-234.

23. Benson JR. Role of transforming growth factor beta in breast carcinogenesis. Lancet Oncol 2004;5:229-239.

24. Hill $M$, Bausero $M$, Mazal $D$, et al. Immunobiological characterization of $\mathrm{N}$-nitrosomethylurea-induced rat breast carcinomas: tumoral IL-10 expression as a possible immune escape mechanism. Breast Cancer Res Treat 2004;84:107-116.

25. Liyanage UK, Moore TT, Joo HG, et al. Prevalence of regulatory $T$ cells is increased in peripheral blood and tumor microenvironment of patients with pancreas or breast adenocarcinoma. J Immunol 2002;169:2756-2761.

26. Singh S, Ross SR, Acena M, et al. Stroma is critical for preventing or permitting immunological destruction of antigenic cancer cells. J Exp Med 1992;175:139-146

27. Spiotto MT, Yu P, Rowley DA, et al. Increasing tumor antigen expression overcomes 'ignorance' to solid tumors via crosspresentation by bone marrow-derived stromal cells. Immunity 2002;17: 737-747.

28. Zou WP. Immunosuppressive networks in the tumour environment and their therapeutic relevance. Nat Rev Cancer 2005;5:263-274.

29. Pollard JW. Tumour-educated macrophages promote tumour progression and metastasis. Nat Rev Cancer 2004;4:71-78.

30. Mahnke K, Ring S, Johnson TS, et al. Induction of immunosuppressive functions of dendritic cells in vivo by CD4 $+\mathrm{CD} 25+$ regulatory T cells: role of B7-H3 expression and antigen presentation. Eur J Immunol 2007;37:2117-2126.

31. Yi $\mathrm{KH}$, Chen L. Fine tuning the immune response through $\mathrm{B} 7-\mathrm{H} 3$ and B7-H4. Immunol Rev 2009;229:145-151.

32. Zhu B, Chen Z, Cheng $X$, et al. Identification of HLA-A*0201-restricted cytotoxic T lymphocyte epitope from TRAG-3 antigen. Clin Cancer Res 2003;9:1850-1857. 